\title{
Monitoring of CRT-D devices during radiation therapy in vitro
}

\author{
Martin Augustynek ${ }^{2 *}$, David Korpas ${ }^{1}$, Marek Penhaker $^{2}$, Jakub Cvek ${ }^{3}$ and Andrea Binarova ${ }^{3}$
}

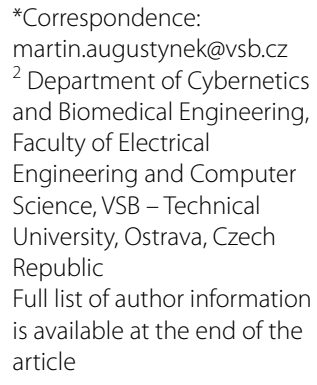

Full list of author information is available at the end of the article

\begin{abstract}
Background: Using of active cardiac medical devices increases steadily. In Europe, there were 183 implants of ICD and 944 implants of PM, 119 of biventricular ICD and 41 of biventricular PM, all per million inhabitants in 2014. Healthcare environments, including radiotherapy treatment rooms, are considered challenging for these implantable devices. Exposure to radiation may cause the device to experience premature elective replacement indicator, decreased pacing amplitude or pacing inhibition, inappropriate shocks or inhibition of tachyarrhythmia therapy and loss of device function. These impacts may be temporary or permanent. The aim of this study was to evaluate the influence of linear accelerator ionizing radiation dose of 10 Gy on the activity of the biventricular cardioverter-defibrillator in different position in radiation beam.

Methods: Two identical wireless communication devices with all three leads were used for the measurement. Both systems were soused into solution saline and exposed in different position in the beam of linear accelerator per $10 \mathrm{~Gy}$ fractions. In comparison of usually used maximum recommended dose of $2 \mathrm{~Gy}$, the radiation doses used in test were five times higher. Using the simultaneous monitoring wireless communication between device and its programmer allowed watching of the devices activities, noise occurrence or drop of biventricular pacing on the programmer screen, observed by local television loop camera.
\end{abstract}

Results: At any device position in radiation beam, there were no influences of the device activity at dose of 10 Gy neither a significant increase of a solution saline temperature in any of the measured positions of CRT-D systems in linear accelerator.

Conclusions: The results of the study indicated, that the recommendation dose for treating the patients with implantable devices are too conservative and the risk of device failure is not so high. The systems can easily withstand the dose fractions of tens Gy, which would allow current single-dose-procedure treatment in radiation therapy. Even though the process of the random alteration of device memory and electrical components by scatter particles not allowed to specify a safe dose during ionizing radiation, this study showed that the safe limit are above the today used dose fractions.

Keywords: Implantable cardioverter defibrillator, Radiation, Interference, Cardiac resynchronization therapy

\section{Background}

Biventricular cardioverter-defibrillators (CRT-D) are life-saving devices that are used in cardiac resynchronization therapy (CRT). CRTs involve the synchronization of both ventricles in order to maintain ventricular contraction towards the septum. This requires 
a permanent pacing using two leads implanted in the right ventricle and in the coronary veins of the left ventricle. CRTs are currently among the standard procedures used for the non-pharmacological treatment of severe heart failure and have been extensively used in recent years in the therapeutic management of patients with end-stage heart failure [1] to increase the ejection fraction. The evidence of the clinical benefit of CRTs offers randomized studies including patients with QRS $>120 \mathrm{~ms}$ as a marker of ventricular dyssynchrony.

The CRT-D implant rate has been steadily increasing worldwide. By conservative estimates, at least one million patients in the USA have permanent artificial cardiac pacemakers [2] and this figure could be in excess of five million patients worldwide [3-5]. These patients may also need to be treated using radiation therapy and any oncology department should expect at least several patients with these devices coming in every year. Radiation therapy is known to have an effect on pacemaker and implantable cardioverter-defibrillator function [6] via electromagnetic interference or through the effects of ionizing radiation [7-11]. The effects of radiation therapy on pacemakers has been studied in detail in the past [11-16]. Based on the studies' results, the American Association of Physicists in Medicine (AAPM) presented recommendations for irradiation of pacemaker patients in 1994 [7] and divided the hazards into two categories-electromagnetic noise interference and radiation damage. The studies mentioned in the AAPM report encompass older pacemaker types based on an old dipole and the first generation of complementary metal oxide semiconductor (CMOS) technology. Modern pacemakers, using newer CMOS circuitry, differ from these devices both in their sensitivity to radiation and in the type of malfunction observed [10]. The effect of irradiation on CMOS technology has also already been described in detail [8].

In the case of implanted cardiac devices, the workflow of treatment planning and delivery is specific for different departments. It is necessary to minimize the dose for devices with aid of contouring as an "organ" at risk. We usually use treatment plans with a minimal number of monitor units and with the shortest treatment time. Pacemaker functioning is checked before and after the first fraction as well as after the radiation course is administered (Fig. 1).

The novelty of our study lies in the unique simultaneous monitoring of the devices during radiation sessions. In previous studies, the devices were just inspected before and after the radiation session by accessing the system, without the possibility of checking the maintaining of the pacing [17-20].

\section{Methods}

To take measurements, two identical biventricular cardioverter-defibrillators, the Cognis model P107 Boston Scientific (Natick, MA, USA) with wireless communication between the device and programmer, were used. Commonly-used leads were attached to the devices: The Fineline Sterox II model 4459 (right atrium port), the Endotak Reliance SG model 0182 (right ventricle and defibrillation ports) and the Acuity Easyaccess model 4554 (left ventricle port). This system was placed into a plastic container containing a saline solution up to $1 \mathrm{~cm}$ above the device. Pacing and defibrillation impedances were measured and were within the manufacturers' required limits. 


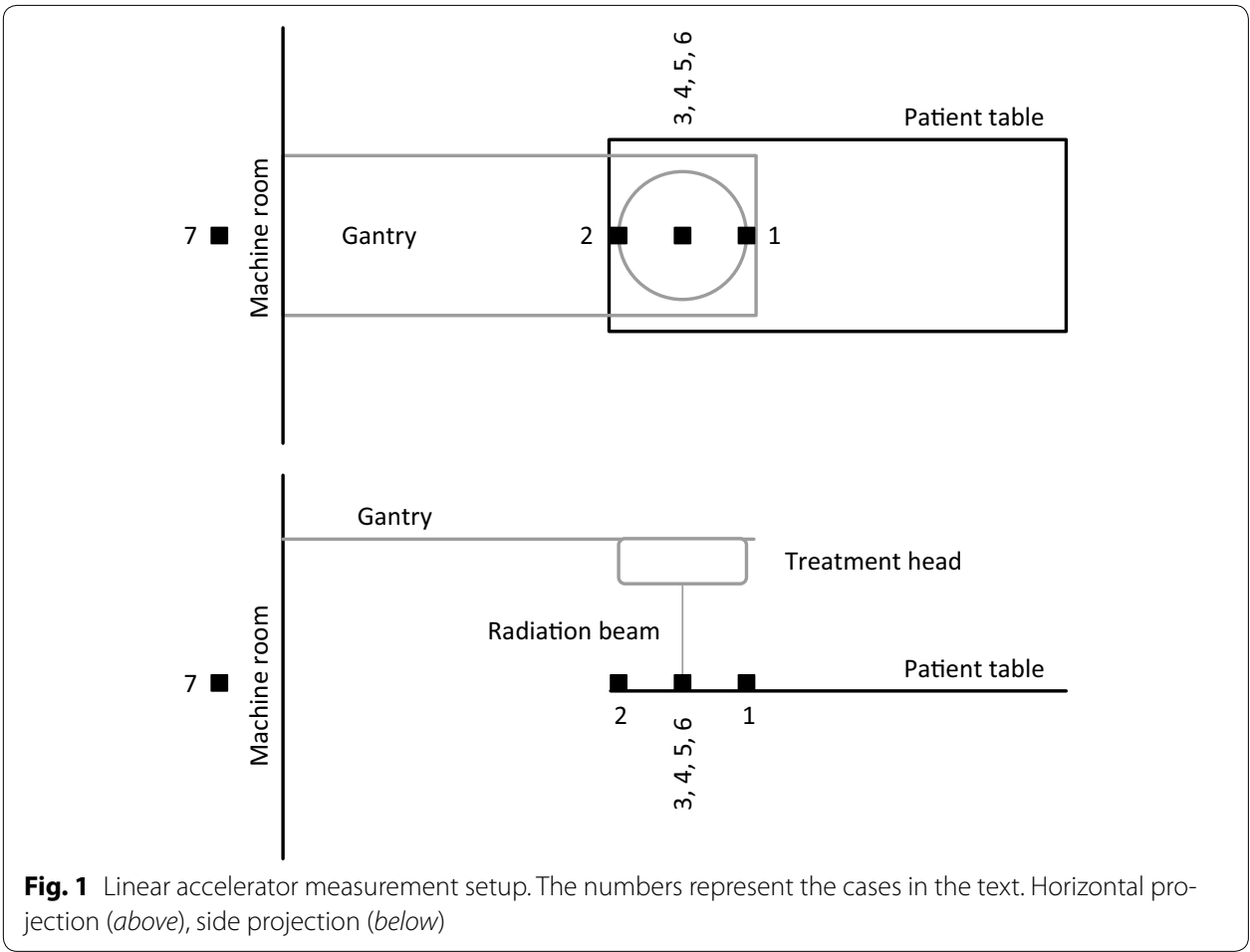

The CRT-D devices were programmed according to current clinical recommendations. The brady pacing mode remained $D D D$ with a Lower Rate Limit of $70 \mathrm{~min}^{-1}$ and Rate Hysteresis off. The output paced the atrium and after $180 \mathrm{~ms}$, it simultaneously paced both ventricles with a Pulse Amplitude of $2 \mathrm{~V}$ for the right atrium and ventricle, a Pulse Amplitude of $4 \mathrm{~V}$ for left ventricle and a Pulse Width of $0.5 \mathrm{~ms}$. The LV pacing vector configuration was LV tip ->LV ring. Tachycardia detection zones were $160 \mathrm{~min}^{-1}$ for VT and $200 \mathrm{~min}^{-1}$. Shock therapy was on. All other parameters were set at nominal values as detailed in Table 1 and all therapies were on.

The type of programmer used was a Boston Scientific (formerly Guidant) Zoom Latitude model 3120 with the ability to wirelessly communicate (ZIP ${ }^{\mathrm{TM}}$ wandless telemetry) with a device. During the trial, the device was interrogated and a programmer screen was placed in front of the camera so that the traces and markers could be watched in the linear accelerator controller room. During radiation exposure, the strip from programmer was printed at a slow speed of $10 \mathrm{~mm} / \mathrm{s}$ to document and check the pacing activity of the device.

An Elekta Precise linear accelerator was used to provide radiation with a photon beam of $6 \mathrm{MeV}$, 1a $0 \times 10 \mathrm{~cm}$ field in aperture, distance to the source of $0.80 \mathrm{~m}$ and a dose rate of $800 \mathrm{cGy} / \mathrm{min}$. This linear accelerator is used for all solid tumors. $6 \mathrm{MeV}$ energy is dedicated mostly for areas cranially from the diaphragm. We measured $30 \mathrm{~cm}$ from the central beam on both sides, on the central beam and in the machine room.

\section{Results}

During the study, we took seven measurements from different positions of the CRT-D can to the central beam of the linear accelerator using different radiation doses (Fig. 1). 
Table 1 Programming of CRT-D devices

\begin{tabular}{ll}
\hline Parameter & CRT-D setting \\
\hline Pacing mode & DDD \\
Lower rate limit & 70 bpm \\
Hysteresis & Off \\
Upper rate & Nominal $130 \mathrm{bpm}$ \\
AV delay & Dynamic 120-180 ms \\
Ventricular pacing & BiV \\
RA, RV sense polarity & bipolar \\
LV pace polarity & bipolar LV tip -> LV ring \\
Rate smoothing & Off \\
VT zone & 160 bpm \\
First VT therapy & Burst: 8 pulses at $81 \%$ \\
& Scan: 8 pulses with 10 ms decr. \\
Second VT therapy & $26 \mathrm{~J}$ \\
Third-last VT therapy & Maximum energy $41 \mathrm{~J}$ \\
VF zone & 200 bpm \\
First VF therapy & $26 \mathrm{~J}$ \\
Second-last VF therapy & Maximum energy $41 \mathrm{~J}$ \\
\hline
\end{tabular}

The CRT-D can with all three leads attached was placed in plastic containers filled with saline solution as was already described with the distance to radiation source of $0.80 \mathrm{~m}$. The field in aperture was $10 \times 10 \mathrm{~cm}$ in all seven cases. The temperature of the saline solution was measured before and after the irradiation of the system. The CRT-D markers and signals were watched using a camera focused on the programmer screen and also printed using an internal programmer printer. With this radiation dose, we did not notice any electromagnetic interference, any drop of biventricular pacing or any other contingencies.

\section{Case 1}

The middle of the CRT-D can was placed $30 \mathrm{~cm}$ from the central beam on the patient deck in the direction from the machine room. The radiation dose was $10 \mathrm{~Gy}$. The temperature of the saline solution remained unchanged at $16^{\circ} \mathrm{C}$.

\section{Case 2}

The middle of the CRT-D can was placed $30 \mathrm{~cm}$ from the central beam on the patient deck in the direction to the machine room. The radiation dose was $10 \mathrm{~Gy}$. The temperature of the saline solution however increased from $16.0^{\circ} \mathrm{C}$ to $17.7^{\circ} \mathrm{C}$.

Case 3

The middle of the CRT-D can was placed on the central beam on the patient deck. The radiation dose was $0.5 \mathrm{~Gy}$. The temperature of the saline solution remained unchanged.

\section{Case 4}

The middle of the CRT-D can was placed on the central beam on the patient deck. The radiation dose was $2 \mathrm{~Gy}$. The temperature of the saline solution however decreased from $17.7^{\circ} \mathrm{C}$ to $17.5^{\circ} \mathrm{C}$. 
Case 5

The middle of the CRT-D can was placed on the central beam on the patient deck. The radiation dose was $10 \mathrm{~Gy}$. The temperature of the saline solution remained unchanged.

Case 6

The middle of the CRT-D can was placed on the central beam on the patient deck. The radiation dose was increased $10 \mathrm{~Gy}$. The temperature of the saline solution remained unchanged.

Case 7

The CRT-D can with the lead system was placed in the machinery room of the linear accelerator. The radiation dose was $10 \mathrm{~Gy}$. The temperature of the saline solution remained unchanged.

\section{Conclusions}

After radiation exposure, the electrical parameters of the pacing system were checked with no significant differences compared to the initial values. Within the devices included in our measurement, there were no observed episodes of device reset, noise sensing, EMI or drop of biventricular pacing with irradiation up to a dose of $10 \mathrm{~Gy}$. Compared to the typically-used maximum recommended dose of $2 \mathrm{~Gy}$, the radiation doses used in our test were five times higher. For the CRT-D devices used in our study, there are no manufacturer's recommendations or guidelines for managing patients undergoing radiotherapy. But with the knowledge that the market available cardiac implantable devices are made from similar components, we can generalize our results to similar CRT-Ds. We limited the results of this measurement to the use of a 6-MV beam with photons as a main component. At higher radiation levels, other effects must be taken into consideration.

Based on recommendations by Guidant from 2003 regarding scatter radiation and ICDs, ICDs may be five to ten times more sensitive to radiation damage than pacemakers since the operating instructions are stored in random access memory that may be more easily damaged by scatter radiation [21]. The best way to safety proceed with radiation therapy for patients with an implanted cardiac device would be simultaneous monitoring on the programmer for devices using wireless communication between the implant and the programmer.

\section{Discussion}

The impact of therapeutic radiation on implanted devices is difficult to predict. Of course, the type of beam is an important consideration. High-energy photon beams have significantly more damaging effects than low-energy beams [22]. Besides this, multiple factors collectively determine the impact of radiation therapy on an implanted device. These factors include the type of implanted device, the distance from the implanted device to the radiation beam, the orientation of the beam to the implanted device, the dose rate and total dose delivered, shielding and the patient's specific anatomy, physiology and heath condition [23]. Due to this variability, it is not possible to specify a safe radiation dosage or guarantee proper device functioning following exposure to ionizing 
radiation. The impact of ionizing radiation will also vary from one pulse generator to another and may range from no changes in function to a loss of pacing and defibrillation therapy. The effects of radiation exposure on an implanted device may remain undetected if patient follow-up is not performed after irradiation. For this reason, clinicians should monitor the device continuously or perform a follow-up right after the radiation session.

Unfortunately, the radiation sensitivity of cardiac implants also seems to be manufacturer dependent. In [10], eleven devices were directly irradiated by $6 \mathrm{mV}$ beams, each to a cumulative dose of 20 Gy. Four of the 11 devices experienced a complete loss of function after only 1.5 Gy of radiation. In our study, we did not see any negative influences up to several tens of Gy. Comparing our results with other older studies, the maximum destructive single dose might vary in a staggering range from 1 up to $80 \mathrm{~Gy}$. It is theoretically possible to assess the risk of hardware circuitry damage. However, random alteration of device memory or electrical components by scatter particles is an issue and it is difficult to predict the safe level. Some devices may be susceptible to other sources of radiation. For example, thermal neutrons can be generated by linear accelerators and they can adversely affect device behavior $[24,25]$.

As it is not possible to absolutely shield the devices, implanted systems will always be somewhat susceptible to the effects of radiation, regardless of any precautions taken. Because most of the implanted devices now are wireless telemetry enabled, our recommendation is to continuously wirelessly monitor the device during radiotherapy sessions.

\section{Authors' contributions}

MA and MP ensured the technical performance of the experiment in a hospital and processed the experimental data, DK performed the pacemaker settings and ensured communication between the device and the programmer and performed data analysis, JC and AB critically revised the manuscript. All authors read and approved the final manuscript.

\section{Author details}

${ }^{1}$ Institute of Nursing, Faculty of Public Policies, Silesian University, Opava, Czech Republic. ${ }^{2}$ Department of Cybernetics and Biomedical Engineering, Faculty of Electrical Engineering and Computer Science, VSB - Technical University, Ostrava, Czech Republic. ${ }^{3}$ Oncology clinic, University Hospital Ostrava, Ostrava, Czech Republic.

\footnotetext{
Authors' informations

D. Korpas was born in Opava, Czechoslovakia, in 1977. He received the M.S. degree in Radioelectronic engineering from Czech Technical University, Prague, in 2001 and the Ph.D. degree in Medical biophysics from Palacky University, Olomouc, in 2004. Since 2004, he has been a biomedical engineer for Boston Scientific Company. He is the author of three books, more than 20 articles, and more than 40 abstracted contributions. His research interests include Active implantable medical devices, Medical devices regulations and Devices for diagnostic and physiological measurement. He is a juridical expert in active implantable medical devices and holds two patents. Mr. Korpas was a recipient of "The best original contribution" of Physician and Technology Journal for 2003 and Dean's price for the best student work for the article "Artery Hemodynamics and Pulse Wave Propagation" in 2004.

M. Augustynek was born in Ostrava, in 1984. He received the M.S. degree in Faculty of Electrical Engineering and Computer science in specialization Measurement and Control in Biomedicine at VŠB - Technical University of Ostrava, Czech Republic, in 2008 and the Ph.D. degree in Technical cybernetics from Technical University of Ostrava, in 2014. Nowadays he is an assistant professor in the Department of Cybernetics and biomedical engineering at the Technical university of Ostrava. He is author or coauthor of six books focused on medical devices and more than 20 original articles. His current research interests are focused on measurement and signal processing of ECG and actimetry, algorithms for pacemakers controlling and algorithms for data processing. His graduation theses "Wireless measuring and visualization of blood pressure" was awarded by the dean and besides he was a grand-aided student of Municipality of Ostrava, Czech Republic.

M. Penhaker was born in Ostrava, in 1972. finished MSc in 1996 at Faculty of Electrical Engineering and Computer science in specialization Measurement and Control in Biomedicine at VSB - Technical University of Ostrava, Czech Republic. He received Ph.D. in Technical Cybernetics in 2000 at VSB - TU Ostrava. In the course of university education he published more than 100 original research articles including over 30 peer reviewed journal papers and also he has more than 30 patents realized. He received several awards, among them the Siemens in "Study of Drive Gear at Mobile Mount with Fuel Cell". His current research interests are focused on sensing, data processing algorithms, instruments for diagnosis and therapy of health corresponding with telemedicine and personal health care.

J. Cvek, (born in 1978) graduated at 3rd Faculty of Medicine, Charles University in Prague (2004) and Faculty of Electrical engineering Czech Technical University in Prague (2002). Ph.D. degree in 2006, consultant in radiation oncology from 2010, two ESTRO (European Society for Radiotherapy and Oncology) certifications in 2005 and 2008. He works as deputy
} 
Head at the Department of Oncology-University Hospital Ostrava (Czech Republic). He is a member of Czech Society for Oncology and Czech Society for Radiation Oncology, Biology and Physics. He has been investigator of three grants in last 5 years. He is co-author of three books and several dozen of contributions have been published in the journal or at the convention. According to the Web of Science eleven citations has been detected. Summary impact factor for 5 years is 10,1. He is interested especially in radiobiology and stereotactic radiotherapy.

A. Binarova was born in Opava in 1985. She graduated from University of Ostrava in 2007 and received bachelor degree in Radiotherapy, Nuclear Medicine and Radio diagnostic Method. After graduation she has started work as RTT in Department of Oncology, in University Hospital Ostrava, and as external lector at Medical Faculty of University of Ostrava. Since 2010 she cooperates with ESTRO and IAEA to establish the national education and research program for RTTs. Also she is an author of Radiotherapy student book, specialized articles and more than 20 abstracted contributions, and she has organized two international courses focused on modern oncological treatment modalities.

\section{Acknowledgements}

This work and the contribution were also supported by the Grant Agency of Czech Republic_GAČR 102/08/1429 project entitled "the safety and security of networked embedded system applications".

The work and the contributions were supported by the SP2015/179'Biomedical Engineering systems XI' project, and this paper was produced within the framework of the project entitled "Support research and development in the MoravianSilesian Region 2014 DT 1—Research Teams" (RRC/07/2014). It was financed from the budget of the Moravian-Silesian Region.

This work was partially supported by the Science and Research Fund 2014 of the Moravia-Silesian Region, Czech Republic.

\section{Competing interests}

The authors declare that they have no competing interests.

Received: 4 December 2015 Accepted: 22 February 2016

Published online: 09 March 2016

\section{References}

1. Bax JJ, et al. Cardiac resynchronization therapy: part 1-issues before device implantation. J Am Coll Cardiol. 2005;46(12):2153-67.

2. Sundar S, Symonds RP, Deehan C. Radiotherapy to patients with artificial cardiac pacemakers. Cancer Treat Rev. 2005:31(6):474-86.

3. Kusumoto FM, Goldschlager N. Cardiac pacing. N Engl J Med. 1996;334(2):89-97.

4. Ector $\mathrm{H}$, et al. The World survey of cardiac pacing and implantable cardioverter defibrillators: calendar year 1997Europe. Pacing Clin Electrophysiol. 2001;24(5):863-8.

5. Bernstein $A D$, Parsonnet $V$. Survey of cardiac pacing and implanted defibrillator practice patterns in the United States in 1997. Pacing Clin Electrophysiol. 2001;24(5):842-55.

6. Kapa S, et al. Effects of scatter radiation on ICD and CRT function. Pacing Clin Electrophysiol. 2008;31(6):727-32.

7. Marbach JR, et al. Management of radiation oncology patients with implanted cardiac pacemakers: report of AAPM Task Group No. 34. American Association of Physicists in Medicine. Med Phys. 1994;21(1):85-90.

8. Last A. Radiotherapy in patients with cardiac pacemakers. Br J Radiol. 1998;71(841):4-10.

9. Calfee RV. Therapeutic radiation and pacemakers. Pacing Clin Electrophysiol. 1982;5(2):160-1.

10. Hurkmans CW, et al. Influence of radiotherapy on the latest generation of implantable cardioverter-defibrillators. Int J Radiat Oncol Biol Phys. 2005;63(1):282-9.

11. Rodriguez F, et al. Radiation-induced effects in multiprogrammable pacemakers and implantable defibrillators. Pacing Clin Electrophysiol. 1991;14(12):2143-53.

12. Adamec $R$, et al. Damaging effect of therapeutic radiation on programmable pacemakers. Pacing Clin Electrophysiol. 1982;5(2):146-50.

13. Venselaar $\mathrm{J}$. The effects of ionizing radiation on eight cardiac pacemakers and the influence of electromagnetic interference from two linear accelerators. Radiother Oncol. 1985;3(1):81-7.

14. Venselaar JL, Van Kerkoerle HL, Vet AJ. Radiation damage to pacemakers from radiotherapy. Pacing Clin Electrophysiol. 1987;10(3 Pt 1):538-42.

15. Salmi J, et al. The influence of electromagnetic interference and ionizing radiation on cardiac pacemakers. Strahlenther Onkol. 1990;166(2):153-6.

16. Ngu SL, et al. Pacemaker function during irradiation: in vivo and in vitro effect. Australas Radiol. 1993;37(1):105-7

17. Uiterwaal $\mathrm{G}$, et al. Interference detection in implantable defibrillators induced by therapeutic radiation therapy. Netherlands Heart J. 2006;14(10):330.

18. Makkar A, et al. Effect of radiation therapy on permanent pacemaker and implantable cardioverter-defibrillator function. Heart Rhythm. 2012;9(12):1964-8.

19. Zaremba T, et al. The effect of radiotherapy beam energy on modern cardiac devices: an in vitro study. Europace. 2014;16(4):612-6.

20. Hashii $\mathrm{H}$, et al. Comparison of the effects of high-energy photon beam irradiation ( 10 and $18 \mathrm{MV}$ ) on 2 types of implantable cardioverter-defibrillators. Int J Radiat Oncol Biol Phys. 2013;85(3):840-5.

21. Guidant Corporation Cardiac Rhythm Management Technical Services. Impact of therapeutic radiation and guidant ICD/CRTD/CRT-P/pacing systems. St. Paul MN, Guidant Corporation, 2004, Revision. pp. 1-6. 
22. Gelblum DY, Amols H. Implanted cardiac defibrillator care in radiation oncology patient population. Int J Radiat Oncol Biol Phys. 2009;73(5):1525-31.

23. Therapeutic radiation and implantable device Systems. 2012, Boston Scientific.

24. Kobayashi $\mathrm{H}$, et al. Soft errors in SRAM devices induced by high energy neutrons, thermal neutrons and alpha particles. in Electron Devices Meeting, 2002. IEDM'02. International. 2002. IEEE.

25. Wilkinson JD, et al. Cancer-radiotherapy equipment as a cause of soft errors in electronic equipment. Device and Materials Reliability, IEEE Transactions on. 2005;5(3):449-51.

Submit your next manuscript to BioMed Central and we will help you at every step:

- We accept pre-submission inquiries

- Our selector tool helps you to find the most relevant journal

- We provide round the clock customer support

- Convenient online submission

- Thorough peer review

- Inclusion in PubMed and all major indexing services

- Maximum visibility for your research

Submit your manuscript at 